\title{
Erratum: Time's Arrow for Shockwaves; Bit-Reversible Lyapunov and "Covariant" Vectors; Symmetry Breaking
}

\author{
Wm.G. Hoover, C.G. Hoover \\ Ruby Valley Research Institute, Highway Contract 60, Box 601 \\ Ruby Valley, Nevada 89833 \\ E-mail: hooverwilliam@yahoo.com
}

Received: 25 July 2013; published online: 31 July 2013

Key words: shockwaves, time reversibility, bit reversibility, Lyapunov instability

A corrected version of Figure 3 appears here. The dashed curve was omitted in the paper version and one of the two electronic versions of our article.

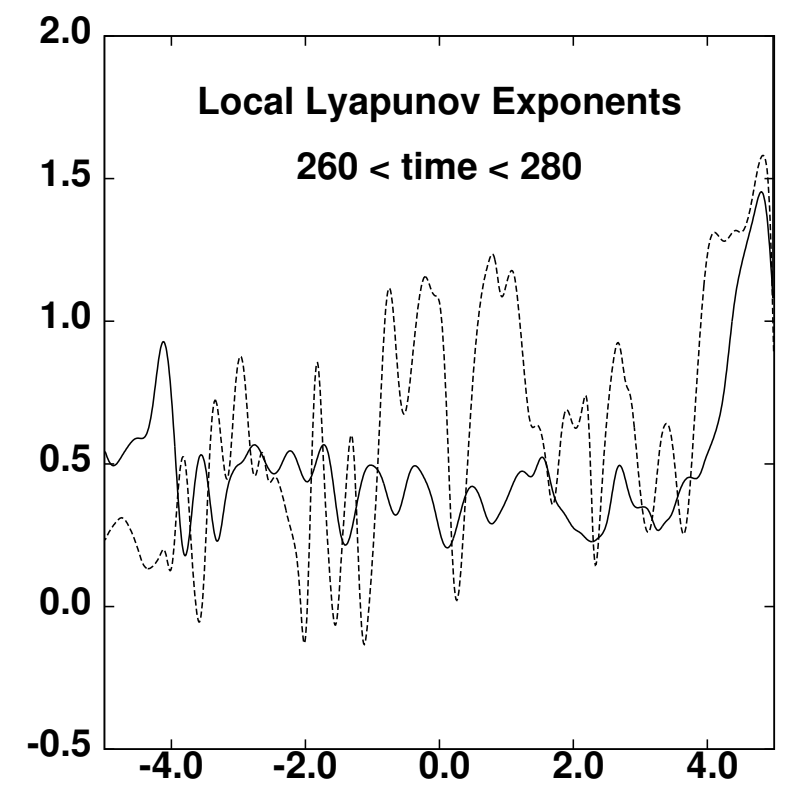

Fig. 3. Largest Lyapunov exponents going forward in time (from 260 to 270 ) and going backward (dashes) in time (from 280 to 270 ) are shown as two curves, at corresponding times. Note that the reversed exponent has brief episodes in which it is negative.
In the original article a sequence of 4000 many-body configurations generated with a bit-reversible leapfrog method, using a timestep $d t=0.01$, and describing the violent collision of two blocks of 800 particles each, was used as a perfectly-reversible "reference" trajectory. The tendency of a nearby Runge-Kutta satellite trajectory to separate from this reference trajectory defines a local Lyapunov exponent for this strongly nonequilibrium system. After four forward and four backward processing steps, reversing the processing direction eight times in all, the nearby satellite trajectory (constrained to remain at a fixed separation from the reference) has converged to machine accuracy, yielding well-defined local Lyapunov exponents both forward in time (full curve) and backward in time (dashed curve). The left-to-right time interval shown in the Figure corresponds to 1000 timesteps, an elapsed time of 10, with the reversal time at the righthand boundary. The right-to-left time interval corresponds to exactly the same configurations, but in reversed order. The time-symmetry-breaking of the local Lyapunov exponents despite the perfect time-reversibility of the reference trajectory was the main point emphasized in both our articles in this issue of CMST. 